\title{
Fluidic low-frequency oscillator consisting of load-switched diverter and a pair of vortex chambers
}

\author{
Václav Tesar̆ $\check{r}^{1}$ Kazimierz Peszynski ${ }^{2}$ Emil Smyk ${ }^{2}$ \\ ${ }^{1}$ Institute of Thermomechanics v.v.i., Academy of Sciences of the CR, Praha, Czech Republic \\ ${ }^{2}$ UTP University of Science and Technology in Bydgoszcz, Faculty of Mechanical Engineering, Control Group, Poland
}

\begin{abstract}
Paper discusses a new configuration of fluidic oscillators, a subject of recent Patent application. There is some similarity with the standard Warren oscillator with its bistable jet-deflection diverter and two feedbacks - which is not suitable for situations demanding very low oscillation frequency. For these conditions the new design replaces jet-deflection switching in the diverter by load-switching effects, with the gradually increased loading by spin-up of fluid in the vortex chambers. The spin-up time also provides the needed time delays. Behaviour is characterised by the oscillation frequency increasing with increasing fluid flow rate - for which was derived a surprisingly simple theoretical solution.
\end{abstract}

\section{Introduction}

Fluidic oscillator discussed in this paper generates selfexited pulsation in fluid flows in its two output terminals. It was developed in a response to demands for very low oscillation frequency that were encountered in generation of gas microbubbles in liquids. Already considerable time ago it has been recognised that the very small bubbles can bring significant advantages in a number of industrial processes (such as, e.g. [1, 2]) - but known methods of the microbubbles generation (such as, e.g., fragmentation by ultrasonics) were all uneconomical. The promising solution found in oscillating the gas flow into a percolation type aerator by inexpensive and reliable fluidic oscillators [2] was unfortunately found not dependable - sometimes it did produce the small bubbles and sometimes not. The hindrance to further progress in this direction was absence of knowledge about the microbubble formation mechanism. Initial approaches were based upon the idea of fragmentation of larger bubbles. If this were really the mechanism, it would be necessary for the fluidic oscillator to operate at very high frequencies. This is obvious since the decomposition into fragments would be the consequence of gradually growing excrescences on the surface of the original larger bubble - and effective formation of the excrescences would naturally require use of higher-mode resonant conditions. Because the "larger" bubbles are in fact quite small and their surface tension forces are consequently large, the resonant frequencies needed for the fragmentation are of the order of $\mathrm{kHz}$ [3]. This is higher than the usual oscillation frequencies of fluidic oscillators [4, 5].
Attempts at achieving the seemingly necessary high frequencies resulted in several extraordinary oscillator designs, like e.g. those described in [6] and [7]. A change in the approach to development was the caused by information obtained in recording the microbubble formations using a high-speed camera and very special optics [8]. The size of the bubbles generated with gas flow oscillations was found to be dictated by phenomenon of repeated conjunctions. The studies described in [8] have also indicated the ways to be followed for suppressing this phenomenon. To the general surprise, the required pulsation frequency was discovered to be the very low, i.e. the very opposite of what used to be believed before. Instead of the high frequencies the search become directed towards obtaining very low frequency.

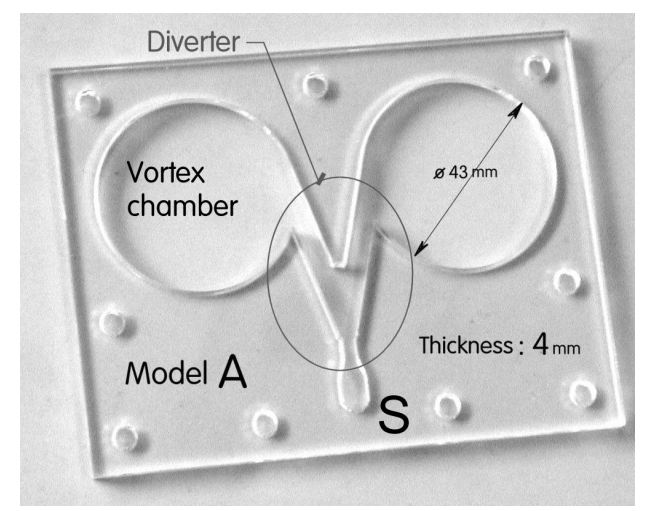

Figure 1. Photograph of the main plate of one of early oscillator models. The load-switched diverter delivers the fluid flow in an alternating manner into one of the two vortex chambers.

\footnotetext{
${ }^{\mathrm{a}}$ Corresponding author: tesar@it.cas.cz
} 


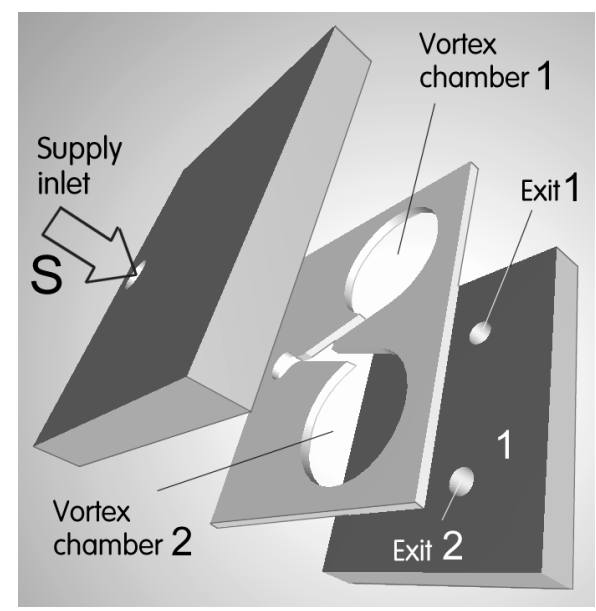

Figure 2. View of the three basic components of authors' oscillator models. The cavities for fluid flow in which is generated the oscillation are in the thin main plate (Fig. 1), clamped between the two thick cover plates, the latter containing the holes for supply (inlet) and outlet flows.

This new direction in the development of the fluidic oscillator encountered several difficulties. It became obvious that standard oscillator designs, which follow the idea of Warren [9], is unsuitable for generation of very low frequencies. The oscillators according to [9] are based on the classical concept of an amplifier with negative feedback. The amplifier is almost always of the bistable fluidic diverter layout, with jet of fluid deflected alternatively to one of two attachment walls by the Coanda effect phenomenon. Each wall leads the jet into one of two output terminals. The deflection is by an action of control flow from one of two control nozzles. This amplifier is converted into the oscillator by providing it with two feedback loop channels connecting the output terminal with the control on the same side nozzle. A part of the fluid flow leaving the amplifier output terminal is fed back into the control nozzle. There it causes separation from the attachment wall and switching of the jet to the opposite side. There the mirror image of the process takes place, resulting in periodic switching. Frequency of oscillation is primarily determined by the length of the feedback channels (which define the necessary time delay between the flow deflection and its return). Secondary influence on the frequency has the supplied fluid flow rate. This dependence on flow is a characteristic property of all no-moving-part oscillatory flows in fluids

This type of oscillator layouts cause no particular problems with standard-sized amplifiers - let us say with supply nozzle widths $\sim 2 \mathrm{~mm}$ - generating oscillation at frequencies from $50 \mathrm{~Hz}$ to $300 \mathrm{~Hz}$. Earlier, where the solution of the microbubble generation problem was sought at high frequencies, development was directed towards minimising the length of the feedback path [6]. Nowadays, with the understanding of the microbubble generating mechanism calling for the very opposite task of obtaining frequencies roughly decimal order of magnitude lower, the obvious approach is to have the feedback channels very long - as long as is physically possible. Typically the lengths are metres or tens of metres.

On one hand, such extreme lengths are difficult to stow in a microbubble generator of reasonable size. More important, on the other hand, is the fact that with such lengths the oscillators tend to stop oscillation. The reason is the hydraulic friction loss in the very long channels and weakening of the feedback signal. The signal may be so weak - and immersed in chaotic turbulence - that is may not suffice for really regular switching.

\section{New layout}

The reason why there is the necessity of large feedback channel lengths is the time delay needed for the deflected jet to move from one attachment wall to the opposite one - and to stay attached there for a part of the oscillation period. Apart from the channel lengths, the parameter that dictates the oscillation frequency is the signal propagation velocity in the channel. Because of the difficulties encountered with the classical layout, it became useful to consider other means of no-movingpart fluidics for obtaining the time delay. Some of the alternative approaches seemed to lead - in principle at least - to more convenient solutions of the stowage problem as well as to elimination of switching irregularity caused by the fluid energy dissipation in the long channels.

An obvious example of an alternative time delay is the accumulation of fluid for a part of the cycle in an accumulation chamber, made possible by fluid compressibility $[10,11]$. An example of a fluidic oscillator using this capacitance mechanism is the Zalmanzon's design, presented in Fig. 4 of ref. [6]. Experience has shown, unfortunately, that this principle of generating time delay is suitable only for high frequency oscillation. Obtaining the desirable long

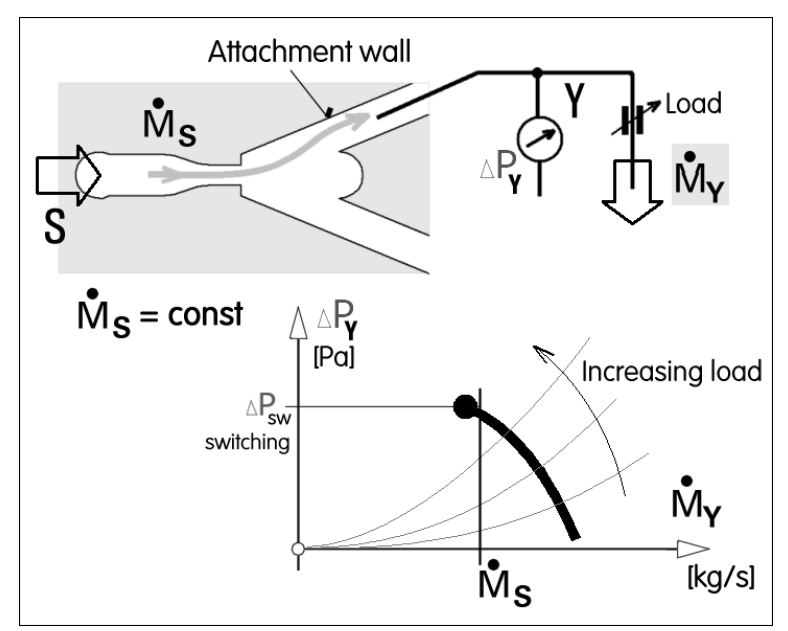

Figure 3. Principle of the load-switching. As dissipance of the load gradually increases the conditions in the output terminal $Y$ move along the loading characteristic curve up to the switching point. - where the Coanda effect can no more keep the flow deflected into the terminal $Y$. 
delay time in this way at the low frequencies of late interest would call for extremely large accumulation chambers. Another alternative solution, one that is discussed in this paper, is the use of the spin-up time of a vortex in a chamber with tangential inlet and centrally located outlet. It takes some time for the rotation to reach its final intensity. The idea of using this mechanism evolved from the use of vortex diodes, first discussed by Zobel [12], in valve-less pumps [13]. Despite the advantage of high achievable steady-state diodity of the Zobel diodes, they were found not suitable for use in microfluidic pumps because of the spin-up and spin-down delays. This made possible operating the vortex diodes only at very low frequencies (and hence with large size of the displacement volumes of the pump). This property, while a drawback in pumping, may be very useful for the here discussed problem of low-frequency oscillators.

The new layout may be explained using the photograph presented in Fig. 1. This oscillator layout is covered by the recent Patent application [14]. In similarity to the Warren's oscillator, the supplied fluid flow enters a diverter valve - with two Coanda-effect attachment walls and corresponding two output channels. Downstream from these diverter outputs are two vortex chambers, each in a configuration closely corresponding to the Zobel [12] diode (with small detail differences demanded my the different manufacturing

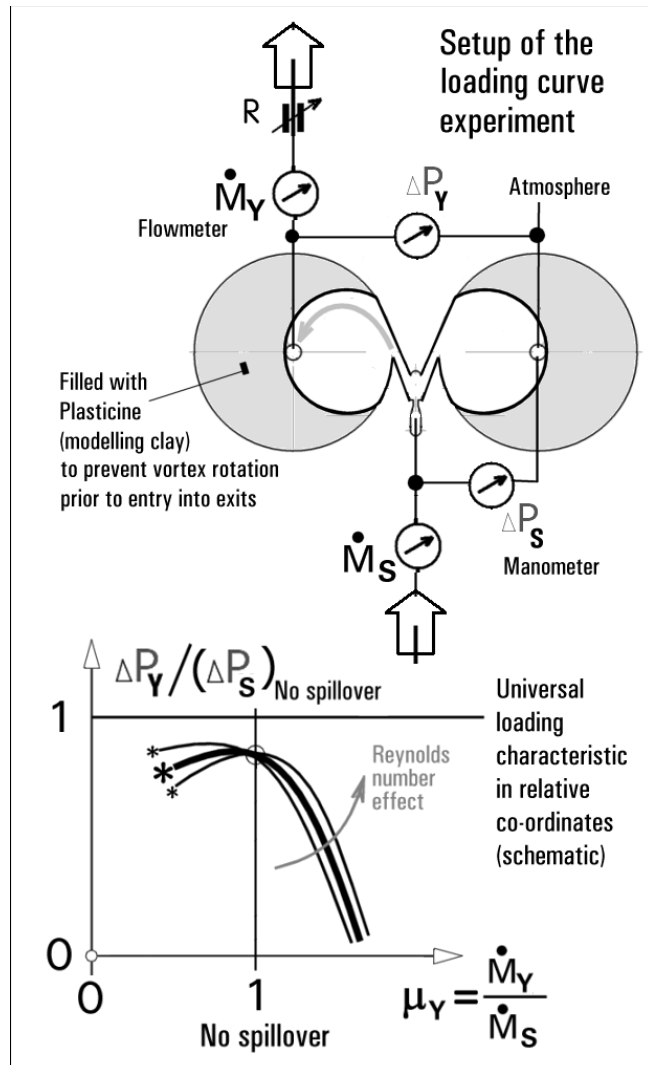

Figure 4. Schematic representation of the setup used in experimental investigation of the loading characteristic. Filling of substantial part of the vortex chambers by Plasticine converted them into settling cavities without centrifugal effects. methods). In principle, it might be possible to switch the diverter by alternating flows issuing from jet-deflecting control nozzles. Nevertheless, in the configuration according to [14] preference is given to a simpler configuration based on the load-switching mechanism.

\section{Load-switching diverter}

The vortex type rotation of fluid takes place in an alternating manner in one of the two vortex chambers. The rotation is caused by the tangential orientation of the chamber entrance from the diverter. Initially, when the flow is switched to enter the vortex chamber, the fluid may is (or may be considered to be) non-rotating - and, hence, the pressure drop across the chamber is relatively low. The diverter thus does not experience any significant resistance to its outflow. The Coandaeffect causing the fluid flow to attach to the active attachment wall in the diverter can easily keep it moving into the load that is here represented by the vortex chamber.

After the initial inertia of the fluid inside the chamber is overcome, the rotation starts and is gradually increasing in intensity. The diverter output flow then meets an increasing resistance. This is represented in the loading characteristic diagram in the bottom part of Fig. 3. On the horizontal co-ordinate in this diagram is plotted the output mass flow rate from the diverter into the location $Y$-- while on the vertical axis is plotted the pressure difference between the location $Y$ and the atmosphere (downstream from the exit from the vortex chamber). Represented in the diagram ion Fig. 3 is the fact that the jet issuing from the supply nozzle entrains fluid flow coming through the inactive output terminal (the bottom one in the picture) - by the jet-pumping effect. As a result, the output flow rate through $Y$ is higher than the supply flow rate through $S$. The thin parabolic lines in the diagram show the characteristics of the load - the vortex chamber. The instantaneous states in the diverter output are determined by the intersections of these load characteristic (thin) and the loading characteristic of the diverter (thick line). As the rotation speed inside the vortex chamber increases, these characteristics are gradually more and more steep. The intersection moves upwards. The diverter has to overcome the higher hydraulic resistance associated with the centrifugal forces. One of the consequences of this process is the decreasing amount of jet-pumped fluid flow. The conditions inside the location $Y$ may, as is the case in Fig. 3, reach the no-spillover regime indicated by the vertical straight line. Further increase of the rotation intensity in the vortex chamber reverses the conditions: instead of the inflow due to the jet pumping, some fluid from the supply terminal $S$ is actually spilled over as $\dot{\mathrm{M}}_{S}-\dot{\mathrm{M}}_{Y}>0$ into the inactive output from the diverter and into atmosphere.

Finally, the conditions reach the state in which the Coanda effect can no more hold the fluid flow attached to the upper (active) attachment wall. This limiting condition is indicated in the diagram in Fig. 3 by the 


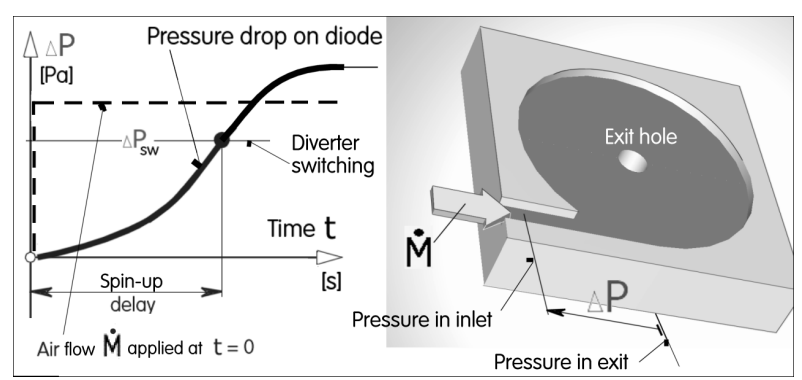

Figure 5. Response of a vortex diode to sudden input of flow $\dot{M}$ into the tangential inlet - after which is this flow kept constant. The vortex spin-up process takes a considerably long time before the pressure difference $\Delta \mathrm{P}$ (between the tangential inlet and central outlet) reaches its final level.

horizontal line - the line of the switching pressure difference. The crossing of this line is marked in the diagram Fig. 3 by a large round black symbol: the fluid flow there separates from the upper attachment wall and is switched to the other, lower wall.

Figure 4 shows schematically the details of the experiments performed for investigating the loadswitching effect of the diverter. Note there the fact that the vortical motion in the vortex chambers in these investigations would distort the diverter behaviour results. To eliminate them, the vortex chambers had to be converted into low flow velocity settling cambers, without the centrifugal effects caused by the vortical rotation. Another difference worth being noted is the fact that instead of the single loading characteristic shown in Fig. 3 were now in Fig. 4 measured three such characteristics, each for a different magnitude of the supply mass flow rate. The graphical presentation in the bottom part of Fig. 4 uses not the absolute co-ordinates (as it does in Fig. 3) but instead relative co-ordinates: on the vertical axis it is the ratio of the output mass flow rate to the supply mass flow rate - while on the vertical co-ordinate are plotted the measured pressure differences between the location $Y$ and the atmosphere, divided by the pressure difference between the supply terminal $\mathrm{S}$ measured in the no-spillover states. Ideally, in these co-ordinates all three loading characteristics might be expected to squeeze into a single universal line (as a consequence of the Eulerian similarity). The

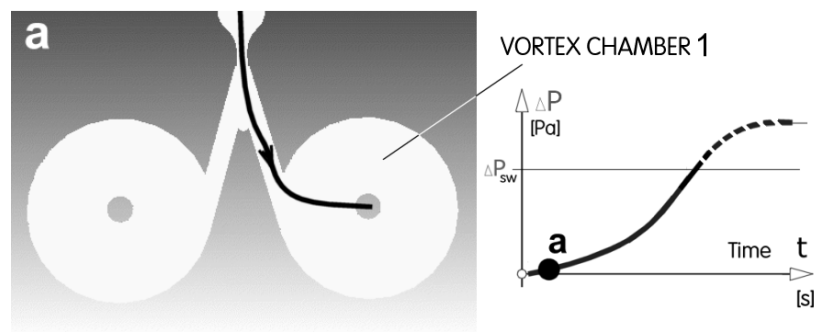

Figure 6. Conditions in the oscillator immediately after the admission of the air flow $\dot{M}$ into the vortex chamber 1. The pressure drop across this chamber is very small since there is no rotation and no centrifugal action to overcome.

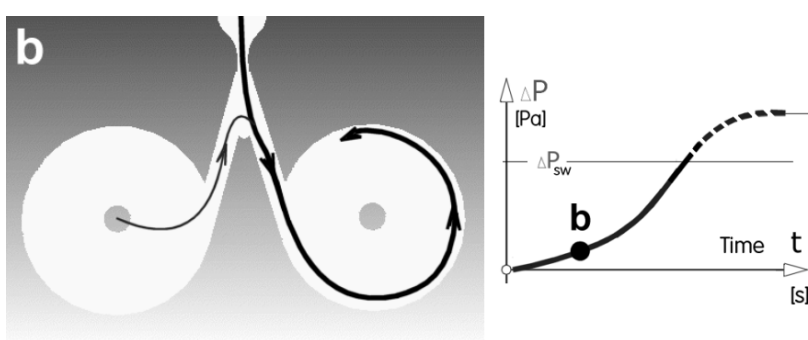

Figure 7. At a later stage "b" than in the previous Fig. 6 the pressure drop across the diode is still small since the spin-up time is quite long. The increased flow rate causes some air to be entrained by jet-pumping effect through the vortex chamber 2 .

characteristics, however, represent among other influences the friction loss component, which is Reynolds-number dependent. Thus the three curves, each measured at different Reynolds number, became slightly dissimilar. Also the position of the loadswitching point is Re-dependent.

\section{Delayed feedback: spin-up}

The switching of the diverter by the increasing load is in the new oscillator made by the increasing pressure drop across a vortex chamber the fluid inside which starts to rotate. At the same time, the inertial response to the starting rotation ensures the desirable time delay.

The vortex chambers are, as is seen in Fig. 5, very simple and therefore easy to manufacture. Their main component is a large circular cavity made in the thin main plate. The rotation is generated by the tangentially directed inlet at the chamber circumference - connected to the exits from the diverter.. In the centre of the vortex chamber is, in the bottom support (cover) plate an exit hole through which the fluid leaves the oscillator.

To get a large time delay it is advisable to make the vortex chamber relatively large (cf. Fig. 1) - because, as is discussed below, the magnitude of the delay effect is directly proportional to the mass of the fluid inside the chamber. Also the very shallow geometry (small vertical difference between the top and bottom cover plates) is desirable. The large exposed cover plate surfaces slow down rapidly by fluid friction the vortical motion once the tangential inflow is switched off. This secures turn-down effect is a necessity because it makes possible starting the spin-up process anew in each

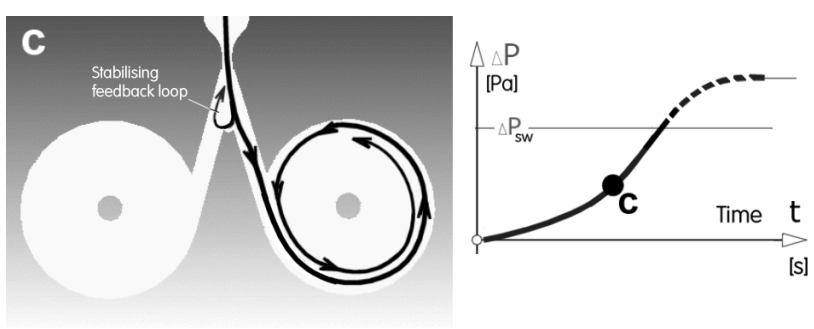

Figure 8. A short time later the rotation in the vortex chamber 1 intensifies. Increased pressure difference to be overcome by the jet causes the jet-pumping to cease. The jet nevertheless remains attached to the $\mathrm{ON}$-side attachment wall. 


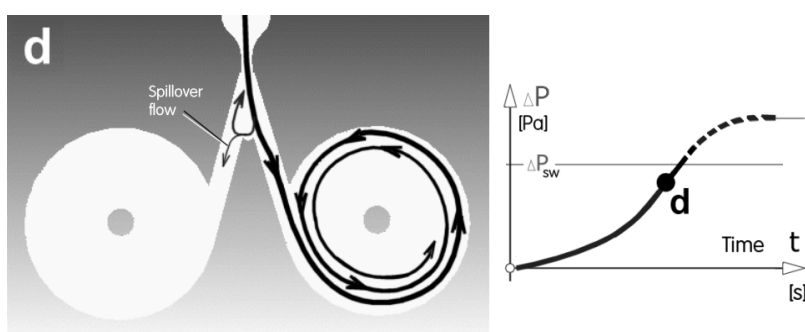

Figure 9. The intensive rotation in the vortex chamber 1 has increased the pressure drop across it substantially. There is some small spillover flow $\dot{M}_{S}-\dot{M}_{Y}>0$ (cf. Fig. 3)

oscillation period (or at least accelerating the rotation if it is not completely stopped by the spin-down effect).

The diagram in the left part of Fig. 5 represents schematically the pressure-drop response of fluid in the vortex chamber to a sudden admission of the constant mass flow rate $\dot{M}$ into the tangential inlet. Important fact presented there is the time delay between the instant of admission and the time at which is reached the diverter switching limit. The pressure response curve in Fig. 5 is drawn as continuing above the diverter switching level. This would be found in experiments with the vortex chamber alone - when the vortex chamber is a part of the oscillator, the switching of the diverter delivers the fluid into the other chamber, as will be schematically presented below in Fig. 15.

Individual phases of the spin-up processes are presented in Figures 6 to 10. As is indicated by the arrows showing the local direction of motion, the processes are complicated by secondary effects - such as the jet-pumping effect that may be present in the very initial stages (Fig. 7) and the stabilising vortical motion created due to the bi-cuspid shape of the splitter nose (note the $3 \mathrm{~mm}$ radius in Fig. 12).

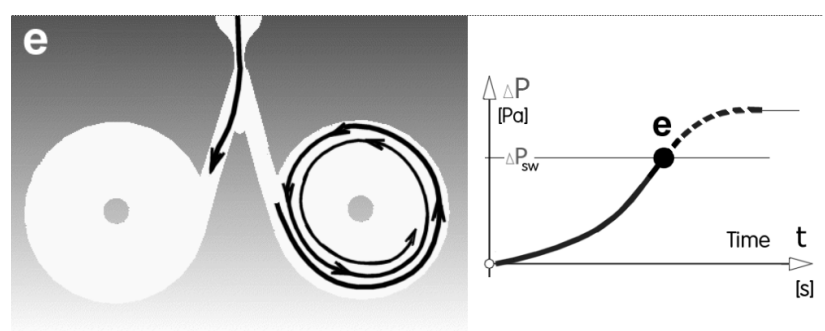

Figure 10. When the load-switching limit is reached, the jet is switched to the other side of the diverter interaction cavity. Due to inertia, some rotation continues in the vortex chamber 1.

\section{Laboratory model}

The model used in the laboratory investigations is shown in the photograph in Fig. 11. It is a larger version among a few initial designs (one ob them the version $A$, is seen in the photograph in Fig. 1). The larges size $B$ and hence low oscillation frequency - was chosen for the tests because of the expected less difficult

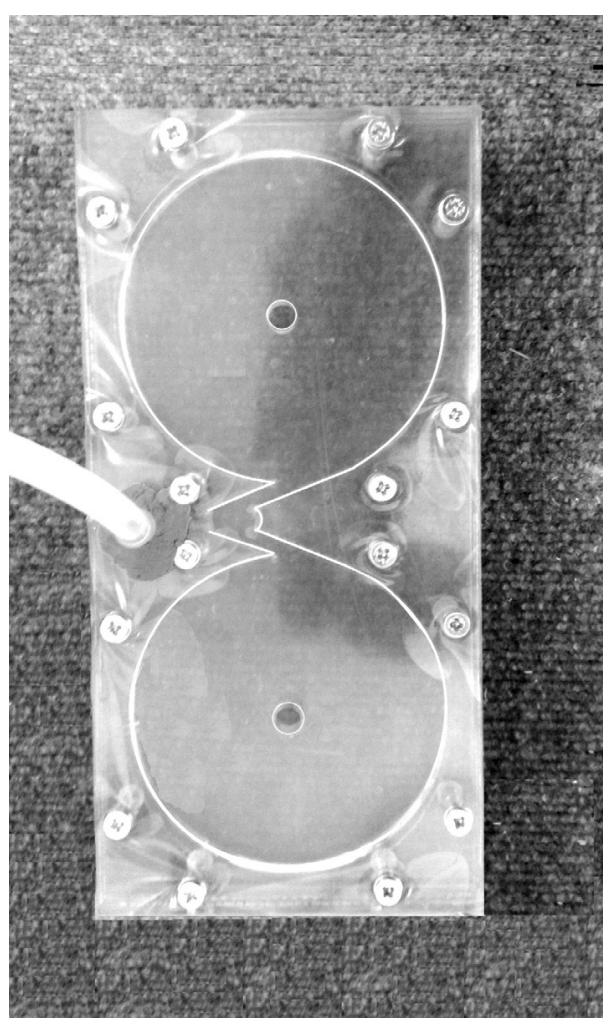

Figure 11. Photograph of the oscillator model taken during test run in the laboratory. Note the sixteen clamping screws and the plastic tube (at left) supplying the air flow. The hotwire anemometer probe (here it was removed and is not seen) was used to measure and record instantaneous velocity samples at one of the vortex chamber exits.

measurements at very low oscillating frequencies. The vortex chamber diameters of the tested model were 100 $\mathrm{mm}$. Geometry of the critical component of the model - the main plate - is presented in Fig. 2 The dominant (i.e. smallest) cross section of the oscillator, the exit of the supply nozzle, was of dimensions $3 \mathrm{~mm} \times 7.8 \mathrm{~mm}$ - the latter dimension being the thickness of the PMMA (polymethylmethacrylate) plate in which the cavities were made by numerically controlled laser cutting. The apex angle of the two attachment walls in the diverter was $45 \mathrm{deg}$ The exist from the vortex chambers were simple holes of $8 \mathrm{~mm}$ diameter.

\section{Measurements and results}

Experiments were performed with air as the working fluid. The supplied air flow rate was measured by digital mass flow meter FMA 1727A — range 0 to 40 standard litres per minute, accuracy $\pm 1.5 \%$ of full scale, with an integral display. The oscillator output was measured by hot-wire MiniCTA anemometer manufactured and supplied by Dantec Dynamics. The probe used was $55 \mathrm{P} 161$, its hot wire positioned downstream from one of the $8 \mathrm{~mm}$ dia. exits from the vortex chamber. The velocities were measured at sampling frequency $2.048 \mathrm{kHz}$, The amplified electric 


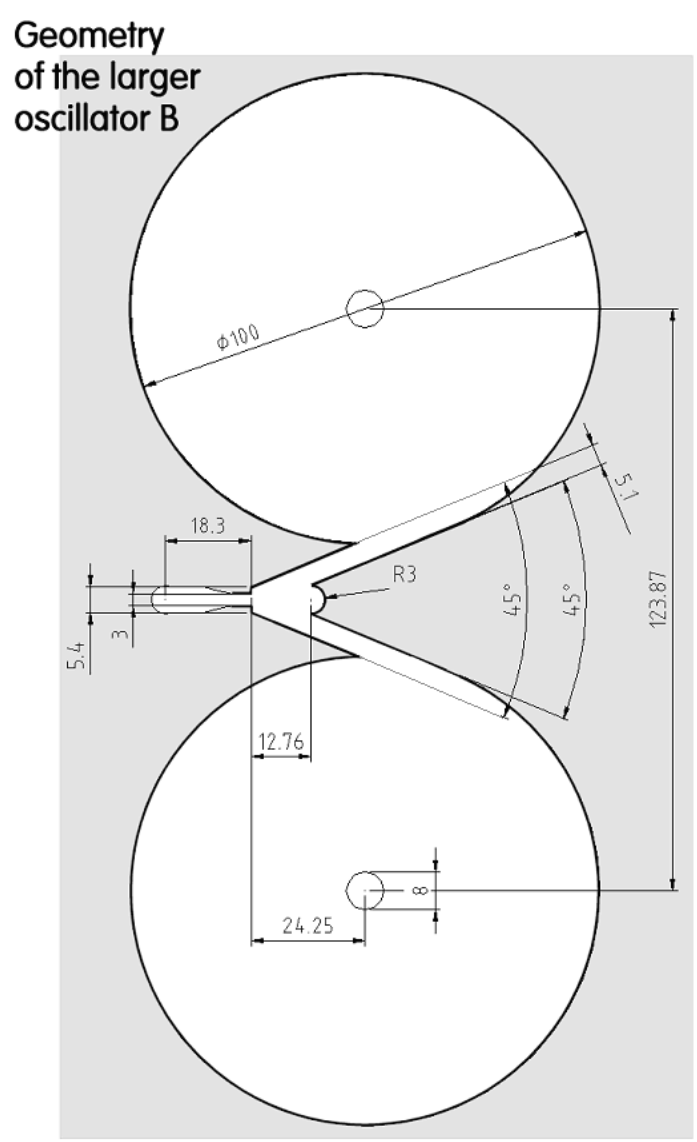

Figure 12. Geometry of the main plate $(7.8 \mathrm{~mm}$ thick) of the oscillator model $\mathrm{B}$ with which were made detailed investigations of oscillatory behaviour.

output from the CTA bridge was linearised and stored in packets, each packed containing 4096 instantaneous velocity data values to be used in subsequent processing. In particular, the data were used for evaluation of time-mean velocity and by computing the velocity spectra by fast Fourier transform.

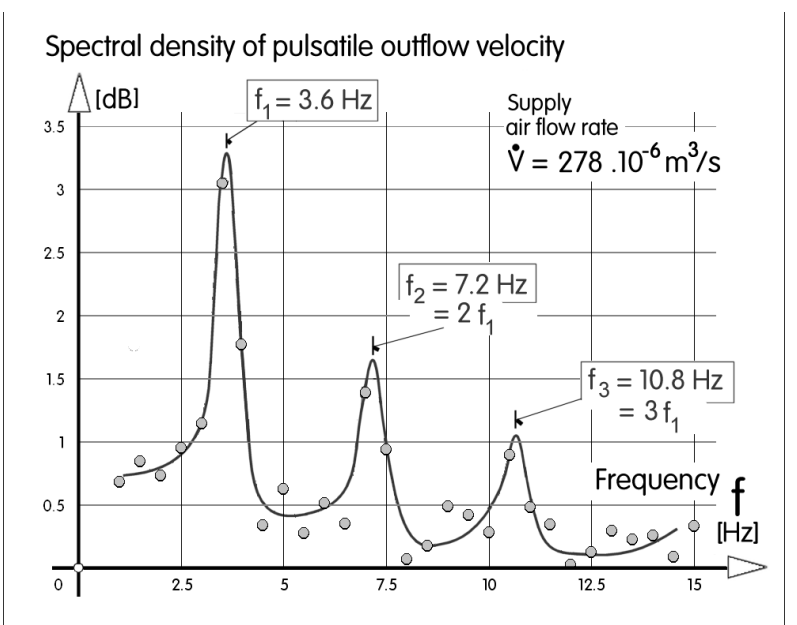

Figure 13. Typical frequency spectrum of the velocity measured at the exit from one vortex chamber at a relatively small air flow rate. Apart from the main frequency $f_{1}$ there are significant higher harmonics.
The next Fig. 13 shows a typical example of the results obtained by the Fourier transformation: a spectral density diagram. This one was obtained at a rather small air flow rate. The most important fact are the really very low frequency levels, which would be difficult to obtain with the usual fluidic oscillators. The main frequency in the case presented in Fig. 13 is mere $\mathrm{f}_{1}=3.6 \mathrm{~Hz}$. Its amplitude is very much higher than all other velocity components, like the quite significant harmonics.

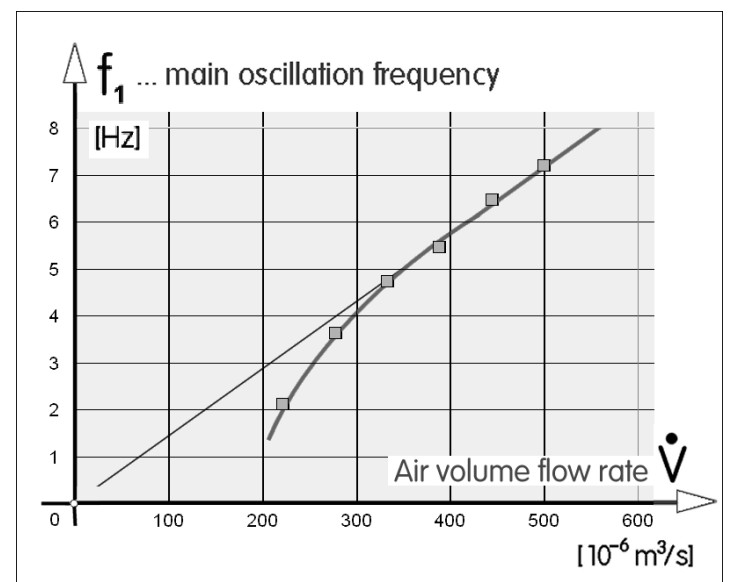

Figure 14. Dependence of the main oscillation frequency $f_{1}$ on the supplied air flow rate. Apart from the deviation at very small flow rates this behaviour indicates constancy of Strouhal number.

Similar diagrams were obtained for 8 other magnitudes of the supply flow rate. In each of them was identified the main frequency $f_{1}$. In the next Fig. 14 is their dependence on the flow rate. It is immediately obvious that the oscillation in the developed oscillator are not a result of acoustic resonance (which would be unlikely anyway because of the size of the resonator needed for so low frequencies) which would be characterised by constant frequency independent on the flow rate. Instead, there is a monotonous growth of the frequency with the increased flow rate. A straight line fitted to the data points above the lower limit volume flow rate $0.310^{-3} \mathrm{~m}^{3} / \mathrm{s}$ passes through the origin of the diagram, which means constancy of the dimensionless parameter known now as Strouhal number Sh.

$$
\mathrm{Sh}=\mathrm{f}_{1} \mathrm{~b} / \mathrm{w}
$$

- a typical property of no-moving-part aerodynamic oscillation associated with shedding of vortices. As usual in fluidics, the Strouhal number in eq. (1) is defined by evaluation from the conditions in the supply nozzle ( - using the supply nozzle width $b=3 \mathrm{~mm}$ and bulk velocity $\mathrm{w}$ ). The values of $\mathrm{w}$ were in the experiments varied between $4.7 \mathrm{~m} / \mathrm{s}$ and $23,7 \mathrm{~m} / \mathrm{s}$. thus the Strouhal number values were all very near to Sh $=0.001$. The corresponding Reynolds number values were in the range from $R e=942.6$ to 4713 . Since the operation of the diverter depends on entrainment of outer air into the jet flow, the nonexistence of the oscillation at velocities lower than the indicated value $w=4.7 \mathrm{~m} / \mathrm{s}$ is obvious the dominating 
laminar character of the jet makes the Coanda effect attachment too weak.

\section{Characteristic time - and a simple theory of oscillator functioning}

There are two parameters of key importance in fluidic oscillators. The first one is the supplied mass flow rate $\dot{M}$ (the volume flow rate, an in Figs. 13 and 14, is an analogous but not conserved parameter, acceptable as alternative in situations with negligible compressibility) while the other one is the frequency of generated oscillation. The latter is in the discussed oscillator principle dependent upon the time delay caused by the spin-up process - and this, in turn, is dependent upon the inertia of the fluid which has to be put into rotation inside the vortex chamber. A very rough approximation providing an information about the magnitude of this inertia is provided by the mass $M_{c h}$, easily evaluated from the diameter $D$ and height $h$ of the chamber as well as the specific volume $v$ of the fluid:

$$
M_{c h}=\frac{\pi D^{2} h}{4 v}
$$

Obviously, the ratio of the two parameters

$$
\mathrm{t}_{\mathrm{c}}=\mathrm{M}_{\mathrm{ch}} / \dot{\mathrm{M}}
$$

is the characteristic time of the spin-up process. It is interesting to compare it with the duration of the oscillation period - or, better, with the half-period

$$
t_{p}=1 /\left(2 f_{1}\right)
$$

- which is the time (compare with the schematic representation of the period in Fig. 15) taken by the pressure difference between the tangential inlet and the central outlet to increase from the initial condition (in Fig. 15 is assumed a condition of completely stopped rotation) to the switching limit. Of course, the spindown time may be different from the spin-up, but this is of no consequence because of the symmetry of the oscillator, whereby the same start-up periods in the vortex chamber 1 and vortex chamber 2 are summed to represent the period.

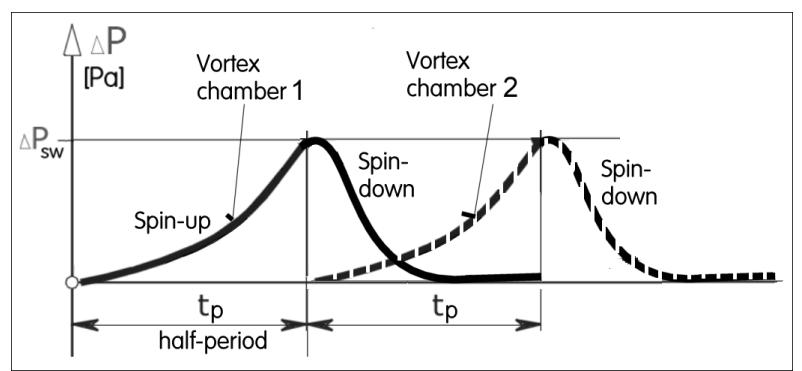

Figure 15. Schematic representation of the mutual relation between the processes in two vortex chambers. The halfperiod lasts $t_{p}=1 /\left(2 f_{1}\right)$

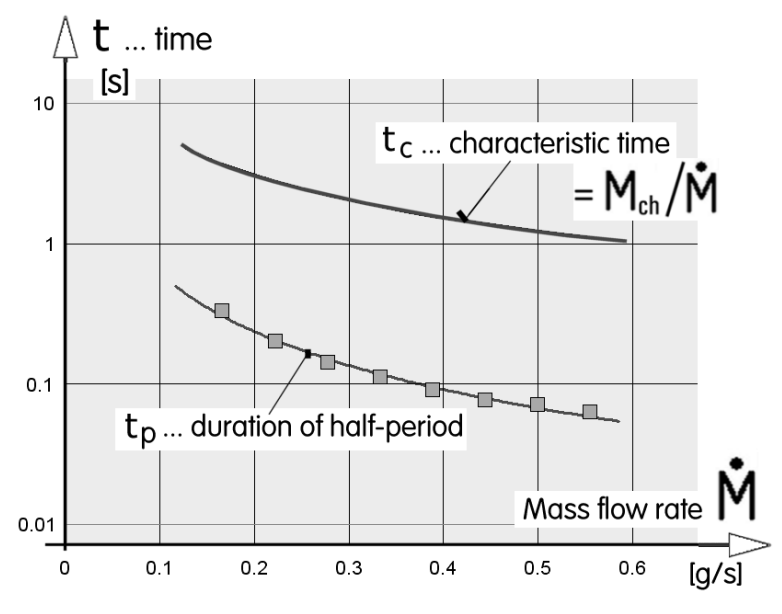

Figure 16. Comparison of the characteristic time (evaluated from the air mass $M_{c h}$ inside the vortex chamber and the supplied air mass flow rate) with the half-period computed from the measured data.

Because the frequency $f_{1}$ in eq. (4) is known from the measurements (Fig. 14), the two elapsed times eq.(3) and eq. (4) may be compared, as is done in Fig. 16. Significantly, while the magnitudes of the two times are different, the character of their dependence on the supplied air mass flow rate is the same. When the characteristic time eq.(3) and the half-period eq. (4) are divided, their ratio was found to be an integer constant

$$
t_{c}=17 t_{p}
$$

- with the exception of the deviation at small flow rates, already discussed as insignificant because of the too low values of Reynolds number. By substitution of eq. (5) and eq. (2) into eq. (3) one obtains a surprisingly simple expression for the oscillator behaviour

$$
f_{1}=\frac{34 v \dot{M}}{\pi D^{2} h}
$$

The importance of such a simple expression is obvious for predicting the performance and designing a system with the oscillator as a component. Of course, the numerical value in the expression eq.(5) is valid only for the same oscillator geometry as presented above in Fig. 12. Perhaps with considerable cautiousness, the results may be extended to slightly different geometries, with different vortex chamber diameters $D$ and different supply nozzle widths $b$ because of the ensuing expression for the Strouhal number - eq. (1)

$$
\mathrm{Sh}=\frac{34 \mathrm{~b}^{2}}{\pi \mathrm{D}^{2}}
$$

- which indeed leads for the geometry from Fig. 12 to the values that were in experiments foud to be all very near to Sh $=0.001$. 


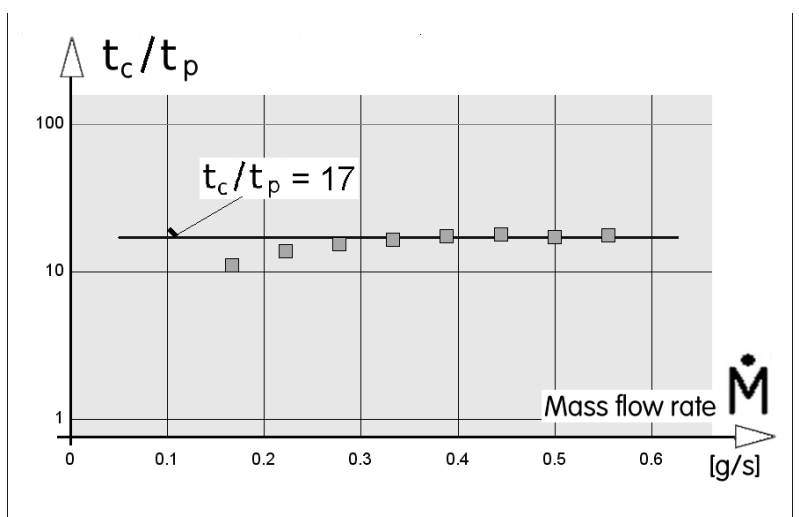

Figure 17. An important fact discovered by analysis of the experimental data: the oscillation period is a simple constant multiple of the characteristic time.

The question about the meaning of the constant 17 in eq. (5) has simple answers. The fact that it is an integer is very probably just an accident. Why it is so large is due to the simplifications introduced in its derivation. It involves the effects of rather complex velocity profiles inside the vortex chamber. Also, it includes the switching time of the diverter (neglected in the derivation completely). Finally it contains the temporal dependences of the secondary flows into (jet pumping) and out from (spillover) the diverter.

\section{Conclusions}

Paper presents interesting results obtained in the development of very-low-frequency fluidic oscillator, originally considered for generation of microbubbles in fluids but obviously suitable for a wide spectrum of other uses. The oscillator is of planar configuration, suitable for modern manufacturing methods. Its main advantage is compactness, which with currently standard oscillators (especially with their necessity of extremely long feedback channels) is a problem.

Apart from demonstration of realisability of the new layout, of considerable value in this paper is the development of a simple and yet very useful theory.

\section{Acknowledgements}

First author obtained support from GAČR - Grant Agency of the Czech Republic, by research grant $\mathrm{Nr}$. 13-23046S. There was also institutional support RVO:61388998.

Third author obtained support from Ministry of Science and Higher Educating of Poland in form of "Diamond Grant" 0201/DIA/2015/44 for young researchers.

\section{References}

1. Microbubbles Revolutionize Industrial Processes, www.mitsubishielectric.com/company/environment/.../ microbubbles/
2. W. J. B. Zimmerman et al., Applied Energy 883357 (2011)

3. V. Tesař, Chemical Engineering Journal. 235. 368 (2014)

4. V. Tesař, S. Zhong, F. Rasheed, AIAA Journal 51397 (2013)

5. Raghu S., Experiments in Fluids 54, 1455 (2013)

6. V. Tesař, Chemical Engineering Research and Design 921603 ( 2014)

7. V. Tesař, Sensors and Actuators A: Physical, 234158 (2015)

8. V. Tesař, Chemical Engineering Science 116849 (2014)

9. R.W. Warren, Negative feedback oscillator, US Patent 3158166 (1962)

10. V. Tesař, Fluidics Quarterly 1177 (1979)

11. V. Tesař, Peszynski K., Pneumatyka 5848 (2006)

12. R. Zobel, Mitt. Hydraulischen Instituts der Technischen Hochschule Munchen, No. 8, R. Oldenbourg, Munich-Berlin (1936).

13. V. Tesař: "Valve-Less Rectification Pumps" Entry on p. 2132 in "Encyclopedia of microfluidics and Nanofluidics", Ed.: Dongqing Li, publ. by Springer Science+Business Media, (2008)

14. V. Tesař, Fluidic oscillator, Czech Republic Patent Application PV 2014-903, December 2014

15. V. Tesař, Proc. of FLUCOME '94, p. 741, Toulouse, France (1994)

16. Section 4.4 in Tesař V., Pressure-driven microfluidics, published by Artech House Inc., Norwood, MA, U.S.A. (2007)

17. V. Tesař, H.C.H. Bandalusena, Experiments in Fluids $\mathbf{5 0} 1225$ (20110

18. V. Tesař, E. Smyk, Chemical Engineering and Processing: Process Intensification 90, 6 (2015)

19. A. Ogawa, H. Murakami H., Internal Flows, P. Doerffler ed., Instytut Maszyn Przeplywowych PAS, Gdansk, Poland, p. 825 (2001)

20. S.-G. Lim, S.-H. Lee, Kim H.-G., Nuclear Engineering and Design 2401139 (2010)

21. J. Yin, L. Jiao, L. Wang, Nuclear Engineering and Design 240970 (2010)

22. A. A. Kulkarni, et al., Chemical Engineering Science 641285 (2009)

23. Jiao., et al., Science China - Technologiocal Sciences 54332 (2011)

24. F. Haakh, Journal of Hydraulic Research 4153 (2003) 